\title{
Profile of Swallowing Disorders in Acute Stroke in Brazzaville
}

 \\ Annette Oball-Mond Mwankie ${ }^{2,3}$, Prince Eliot Sounga Bandzouzi ${ }^{2,4}$, \\ Dinah Happhia Motoula Latou ${ }^{1,2}$, Bébène Bandzouzi Ndamba ${ }^{1,2}$ \\ ${ }^{1}$ Department of Neurology, University Hospital of Brazzaville, Brazzaville, Republic of Congo \\ ${ }^{2}$ Marien NGOUABI University of Brazzaville, Faculty of Health Sciences, Brazzaville, Republic of Congo \\ ${ }^{3}$ Stomatology and Maxillofacial Surgery Department, University Hospital of Brazzaville, Brazzaville, Republic of Congo \\ ${ }^{4}$ Neurology Department, Loandjili General Hospital, Pointe Noire, Republic of Congo
}

Email address:

mgaetimm@yahoo.fr (G. A. Mpandzou)

${ }^{*}$ Corresponding author

\section{To cite this article:}

Ghislain Armel Mpandzou, Paul Macaire Ossou-Nguiet, Lopresty Luberde Ngouala, Annette Oball-Mond Mwankie, Prince Eliot Sounga Bandzouzi, Dinah Happhia Motoula Latou, Bébène Bandzouzi Ndamba. Profile of Swallowing Disorders in Acute Stroke in Brazzaville. Clinical Neurology and Neuroscience. Vol. 3, No. 1, 2019, pp. 16-23. doi: 10.11648/j.cnn.20190301.14

Received: March 14, 2019; Accepted: May 8, 2019; Published: May 30, 2019

\begin{abstract}
Swallowing disorders are common in stroke and cause significant morbidity and mortality. The aim of this study was to determinate the frequency of swallowing disorders and its impact on the prognosis of stroke, as well as the contribution of the local feeding protocol at University Hospital of Brazzaville. An interventional study was carried out between March and August 2016 in the department of neurology, with a follow-up of three months. It included all patients hospitalized for stroke and swallowing disorders. If necessary, a nasogastric tube was placed and local feeding protocol was initiated. Among 219 patients admitted for stroke, 59 (26.9\%) had swallowing disorders. The DePippo test was positive in 54 (91.5\%) patients. The mean age of the patients was $69.1 \pm 13.8$ years with a sex ratio of 1.3 . Cough during feeding (79.7\%) and swallowing effort $(81.4 \%)$ were the most common complaints. The majority $(72.9 \%)$ of patients had bilateral pyramidal involvement, and 23 $(39 \%)$ a history of stroke. The local feeding protocol was respected by only half of patients, without any impact on the occurrence of malnutrition and dehydration (respectively, $\mathrm{p}=0.58$ and $\mathrm{p}=0.79)$. Death was observed in $32(54.24 \%)$ patients primarily for bronchopneumopathy $(n=5,15.62 \%)$, false roads $(n=4,12.5 \%)$ and cerebral hematoma $(n=4,12.50 \%)$. Eleven $(34.4 \%)$ patients died at home for an undetermined cause. Detection and appropriate management of swallowing disorders in acute stroke, must be systematic and included in management protocols of stroke.
\end{abstract}

Keywords: Stroke, Swallowing Disorders, Feeding Protocol

\section{Introduction}

Stroke is defined as a sudden focal neurological deficit secondary to cerebral parenchymal injury caused by infarction or hemorrhage [1]. Its prevalence is estimated at 317.3 (314.0-748.2)/100,000 population in Africa, with particularity a high frequency of hemorrhagic stroke in most countries $[2,3]$. Stroke is the main cause of hospitalization and death in neurology department at the University Hospital of Brazzaville [4]. Swallowing disorders are a complication of stroke, with an incidence of 40 to $80 \%$ in the acute stroke, and associated with high morbidity and mortality [5-11]. Although about $50 \%$ of patients spontaneously recover swallowing function in the first two weeks, their presence initially exposes patients to multiple complications and comorbidities, i.e. bronchopneumopathies, fatigue, malnutrition, dehydration $[12,13]$. Swallowing disorders also interfere with the recovery of motor function and cause functional discomfort disrupting daily life [6]. Their presence is associated with poor prognosis $[14,15]$. Early detection of swallowing disorders and nasogastric tube placement with a well-defined feeding protocol would prevent the occurrence 
of these various complications, thereby reducing short-term and intermediate-term stroke morbidity and mortality [15, 16]. A recent review showed that preventive antibiotics within 48 hours after acute stroke in patients with swallowing disorders will reduce the occurrence of bronchopneumopathies [17]. Furthermore, several studies currently show the efficacy and safety of telemedicine in assessing swallowing disorders and improving adherence to rehabilitation. Which could have an interest in our context where access to care is limited and the number of speech therapists [18, 19]. Recently, brain functional magnetic resonance imaging study demonstrated that both hemispheric and brainstem stroke in patients with swallowing disorders showed an improvement after swallowing training [20].

In Africa, as in Congo, few studies have focused on the outcome and management of swallowing disorders after stroke, including the contribution of a local protocol nasogastric feeding. The purpose of this work was to establish the evolution profile of post-stroke swallowing disorders in our hospital.

\section{Patients and Method}

This is an interventional study conducted in the Department of neurology and Medical Emergencies, of University hospital of Brazzaville, from March $1^{\text {st }}$ to August $1^{\text {st }}, 2016$. The Department of neurology has a nutritionist but no speech therapist. We included in the study, all patient with swallowing disorders, admitted for stroke documented by imaging (CT or MRI brain) and have given their informed consent themselves or default by a third party informing. Exclusion criteria were; the initial presence of a deep coma (Glasgow score less than 9), the presence of a history of cancer or any other otorhinolaryngological pathology, the existence of swallowing disorders in relation to anterior stroke or a neurological pathology other than vascular. Only patients with withdrawn consent after inclusion were excluded.

The presence of swallowing disorders was objectified by looking for the gag reflex by a daily clinical examination during the acute phase of stroke and at each stage of followup. This research consisted in stimulating the base of the tongue or the posterior pharyngeal wall with a tongue depressor in search of a reduction or an abolition of the reflex. The $3 \mathrm{Oz}$ water swallow test of DePippo was performed and compared to the tongue-depressed exam [21]. The DePpipo test consists in having the patient continuously drink 5-10, 45 and then $90 \mathrm{ml}$ of water. It is positive when the patient coughs into minute after taking drink or when the voice becomes gurgling, wet or hoarse. Once the diagnosis of swallowing disorders was established, a nasogastric tube was placed based on patient consent and swallowing disorders severity. Interest and usage terms of the nasogastric tube had been previously explained to the patient and patient care. A standardized low-cost local feeding protocol (Table 1) was then introduced. This protocol includes meals and snacks available locally with a schedule of feeding schedules.

Table 1. Local feeding protocol.

\begin{tabular}{|c|c|c|}
\hline Hours & Quantity & Composition \\
\hline 7:00 & Snack (100mL mixed or pressed fruit juice) & Fruit juice \\
\hline $8: 00$ & Meals ( 2 to 3 syringes of $50 \mathrm{~mL}$ ) & Vegetable broth + Cereals/Legumes + Sugar + Salt + Egg white \\
\hline 10:00 & Snack ( 1 to 2 syringes of $50 \mathrm{~mL}$ as needed) & Water or Oral Rehydration Solution (ORS) \\
\hline $12: 00$ & Meals ( 2 to 3 syringes of $50 \mathrm{~mL}$ ) & Vegetable broth + Cereals/Legumes + Sugar + Salt + Smoked fish $/$ Caterpillars $/$ Peanut paste \\
\hline 13:00 & Snack $(125 \mathrm{~mL}$ natural yoghurt $+100 \mathrm{~mL}$ water $)$ & Natural yogurt + Water \\
\hline $16: 00$ & Snack (100mL mixed or pressed fruit juice) & Fruit juice \\
\hline 18:00 & Meals ( 2 to 3 syringes of $50 \mathrm{~mL}$ ) & Vegetable broth + Cereals $/$ Legumes + Sugar + Salt + Mixture local vegetables \\
\hline 20:00 & Snack (100mL of beverage of choice) & Water, ORS, Natural yoghurt, Fruit juice \\
\hline
\end{tabular}

When the patient is clinically stabilized, home hospitalization with an indwelling nasogastric tube is recommended when swallowing disorders persist. At home, the nasogastric tube was changed every two weeks to one month by a medical service.

The variables studied included age, occupation (unemployed, informal sector, public official category 1, 2 or 3: "respectively for a monthly income (in CFA) > 300000 , between 100000 and 300000 or $<100000$ ", or private sector), level of education, marital status, history of active smoking and/or alcohol intake, history of diabetes mellitus, hypertension and/or stroke prior.

Clinically, there was the presence of hemiplegia or paresis and its laterality, the presence of a bilateral pyramidal syndrome, cough and/or difficulty breathing during the meal, saliva accumulation in the mouth outside of meals, notion of swallowing effort, time to diagnose swallowing disorders and to the placement of nasogastric tube after stroke onset, presence of dehydration, malnutrition and/or other complications. The brain imaging data related to the type of stroke, ischemic or hemorrhagic. The study was approved by the Ethics Committee for Health Sciences Research.

We used Microsoft Excel 2010 software for the design of database. Quantitative variables were expressed as mean \pm standard deviation and categorical variables in terms of size and percentage. The comparisons between variables were made by the Pearson Chi-2 test or Fisher exact test under SPSS 20.0. The threshold of significance was set at 0.05.

\section{Results}

Among 219 patients admitted for stroke during the study period, 59 (26.9\%) had swallowing disorders. The DePippo test was positive in $91.5 \%(n=54)$ of patients after ingestion of $90 \mathrm{ml}$ of water. After ingestion of 5-10 and $45 \mathrm{ml}$ of water, this test was positive, respectively in 30 (45.8\%) and 24 
(40.7\%) patients. The mean time to diagnosis swallowing disorders in relation to the occurrence of stroke was $6.2 \pm 6$ days [ 1 to 30 days]. No patient was lost sight nor withdrew from the study.

There were $33(55.9 \%)$ men and $26(44.1 \%)$ women (sex ratio: 1.3 ), with a mean age of $69.1 \pm 13.8$ years [36 to 95 years]. Sociodemographic characteristics and anamnestic, clinical and paraclinical data are presented in Tables 2 and 3.

Table 2. Socio-demographic characteristics.

\begin{tabular}{lll}
\hline & Effective (n) & Frequency (\%) \\
\hline Level of education & & \\
\hline Unschooled & 16 & 27.1 \\
Primary & 9 & 15.3 \\
Lower secondary & 14 & 23.7 \\
Graduation & 10 & 16.9 \\
Academic & 10 & 16.9 \\
\hline & & Frequency (\%) \\
\hline & Effective (n) & \\
\hline Professional status & & 18.6 \\
\hline Unemployed & 11 & 18.6 \\
Public service category $1 *$ & 11 & 3.4 \\
Public service category $2 *$ & 2 & 28.9 \\
Public service category $3 *$ & 17 & 5.1 \\
Private sector & 3 & 25.4 \\
Informal sector & 15 & \\
\hline & & Frequency (\%) \\
\hline & Effective (n) \\
\hline Marital status & 21 & 35.6 \\
\hline Single & 8 & 13.6 \\
Free union & 19 & 32.2 \\
Married & 3 & 13.6 \\
Divorced & 8 & \\
Widower & & \\
\hline
\end{tabular}

*Public service category 1, 2 et 3 "monthly income (in CFA) respectively: > 300,000 , between 100,000 and 300,000 and $<100,000$ ".
Table 3. Anamnestic, clinical and paraclinical data.

\begin{tabular}{lll}
\hline & Effective (n) & Frequency (\%) \\
\hline History of hypertension & 45 & 76.3 \\
History of diabetes & 9 & 15.3 \\
History of alcoholism & 16 & 27.1 \\
History of active smoking & 2 & 3.4 \\
History of stroke & 20 & 33.9 \\
Right hemiplegia & 11 & 18.6 \\
Left hemiplegia & 4 & 6.8 \\
Bilateral pyramidal syndrome & 43 & 72.9 \\
Cough during feeding & 47 & 79.7 \\
Breathing difficulty during feeding & 3 & 5.1 \\
Accumulation of salivate outside of & 13 & 22 \\
meals & 48 & 81.4 \\
Swallowing effort & 15 & 25.4 \\
Hemorrhagic stroke & 44 & 74.6 \\
Ischemic stroke & & \\
\hline
\end{tabular}

The nasogastric tube was placed in $91.5 \%(n=54)$ of the patients. The mean time to place nasogastric tube in relation to the diagnosis of swallowing disorders was $1.35 \pm 1$ day [0 to 7 days].

Among family carers, the rate of adhesion to the nasogastric tube was $89.8 \%(n=53)$. During the period of hospitalization among the 54 patients with nasogastric tube, the local feeding protocol was correctly observed in 27 (50\%) patients. Five $(8.5 \%)$ patients did not have a nasogastric tube because their swallowing disorders were minimal. The return at home was recommended towards the second month of hospitalization.

During follow-up, swallowing disorders regressed in 12 $(20.3 \%)$ patients and persisted in $13(22 \%)$ others. Table 4 shows the chronological evolution of swallowing disorders during the three months of follow-up.

Table 4. Evolution of swallowing disorders over three months of follow-up.

\begin{tabular}{|c|c|c|c|c|c|}
\hline & D7 $(n=59)$ & D14 $(n=50)$ & M1 $(n=38)$ & M2 $(n=35)$ & M3 $(n=15)$ \\
\hline & n (\%) & n (\%) & n (\%) & n (\%) & n (\%) \\
\hline Recovery & $2(3.4)$ & $6(12)$ & $1(2.6)$ & $4(11.4)$ & - \\
\hline Stationary & $31(52.5)$ & $36(36)$ & $28(73.7)$ & - & $13(86.7)$ \\
\hline Complications & $19(32.2)$ & $20(40)$ & $7(18.4)$ & $15(42.9)$ & $1(6.7)$ \\
\hline Death & $7(11.9)$ & $6(12)$ & $2(5.3)$ & $16(45.7)$ & $1(6.7)$ \\
\hline
\end{tabular}

$\mathrm{N}^{\circ}$ effective, \%: percentage, $\mathrm{D}$ : day, $\mathrm{M}$ : month.

Complications observed in relation with swallowing disorders were dehydration $(n=15,25.4 \%)$, malnutrition $(n=6,10.2 \%)$, false roads $(n=4,6.8 \%)$, bronchopneumopathy $(\mathrm{n}=5,8.5 \%)$. Among patients who developed complications during follow-up, there were five patients without nasogastric tube and $25(92.6 \%)$ of 27 patients who have not respected the local feeding protocol. The comparison between the occurrence of dehydration and malnutrition according to the respect or not of the local feeding protocol is shown in Table 5.

Table 5. Link between respecting local feeding protocol and the occurrence or not of malnutrition and dehydration.

\begin{tabular}{|c|c|c|c|c|c|c|c|}
\hline & & \multicolumn{2}{|c|}{$\begin{array}{l}\text { Local feeding protocol } \\
\text { respected }(n=27)\end{array}$} & \multicolumn{2}{|c|}{$\begin{array}{l}\text { Local feeding protocol not respected }+ \\
\text { patients without nasogastric tube }(n=27)\end{array}$} & \multirow[t]{2}{*}{ OR $(95 \%$ CI $)$} & \multirow[t]{2}{*}{$\mathbf{p}$} \\
\hline & & $\mathbf{n}$ & $\%$ & $\mathbf{n}$ & $\%$ & & \\
\hline Malnutrition & yes & 2 & 7.4 & 3 & 11.1 & $0.77(0.08-5.61)$ & 0.58 \\
\hline Dehydration & yes & 4 & 14.8 & 8 & 29.6 & $0.52(0.12-1.99)$ & 0.79 \\
\hline
\end{tabular}

$\mathrm{n}$ : effective, \%: percentage, OR: odds ratio, CI: confidence interval, p: p-value à 0,05 .

Thirty-two (54.2\%) patients died during follow-up, including eleven (34.4\%) after returning home. A comparison of 
anamnestic, clinical, imaging and evolutionary data between surviving and deceased patients is presented in Table 6. Of these patients, five (15.6\%) did not have a nasogastric tube and $23(71.9 \%)$ did not follow the local feeding protocol. The causes of death are presented in Figure 1.

Table 6. Comparison of anamnestic, clinical and imaging between survivors and deceased patients.



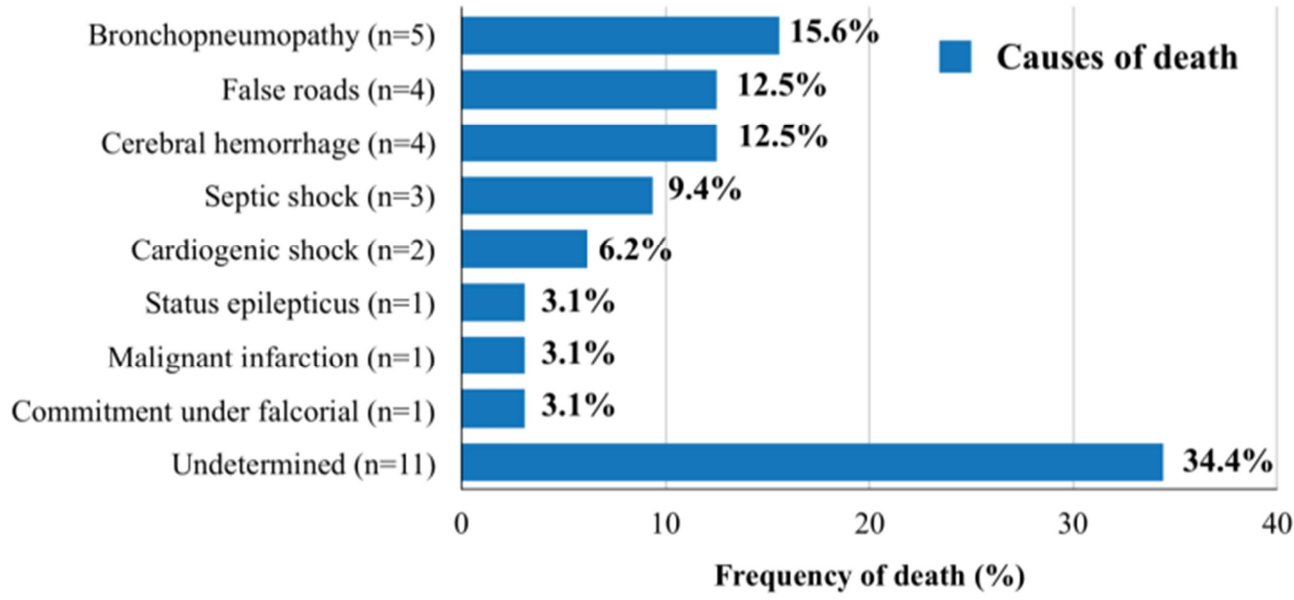

Figure 1. Causes of death.

\section{Discussion}

\subsection{Diagnosis of Swallowing Disorders}

The diagnosis of swallowing disorders in our study was established by the search for gag reflex, which compared to the Deppipo test, showed a nearly similar prevalence of swallowing disorders (respectively in 59 and 54, patients). The DePippo test has a sensitivity in order of 76 to $94 \%$, which makes our diagnostic criteria more reliable [21].

The frequency of swallowing disorders in our study was $26.9 \%$. In the literature, it is highly variable, from $20 \%$ to $65 \%$, and is generally explained by the difference in diagnostic methods, especially the frequent use of videofluoroscopy or videoendoscopy in developed countries [22-28]. Crary et al. has indicated interest for of evaluating swallowing rate per minute in the diagnosis of post-stroke swallowing disorders, with a sensitivity of $96 \%$ and a specificity of $68 \%$, compared to other methods [29].
The time to onset swallowing disorders in our patients was $6.16 \pm 6$ days. This time in stroke is most often 3 to 4 days, and the incidence of swallowing disorders would increase after $24 \mathrm{~h}$ of admission [29-32].

\subsection{Sociodemographic Characteristics}

As in most studies, the mean age of our patients was high at $69.1 \pm 13.8$ years $[22,28,33]$. The frequency of swallowing disorders in elderly patients is well known, due to neurological or otorhinolaryngological affections $[28,32,34-37]$. The male predominance of our patients is explained by the more frequent consumption of tobacco and alcohol by men in the Congo, as a risk factor for stroke [38]. A male predominance of stroke is often reported [13, 38-40]. In contrast, Alancar Nunes et al. report a female predominance in 30 patients with swallowing disorders, as well as Paciaroni et al. in 141 patients with swallowing disorders $[22,33]$. 


\subsection{Cardiovascular Risk Factors}

High blood pressure, prevalent in our population, is in fact the main risk factor for stroke in Congo. More than $60 \%$ of cerebrovascular disorders are due to high blood pressure [4, 41]. However, Arnold et al. found a significantly lower prevalence of hypertension $(36.2 \%)$ in patients with versus without swallowing disorders $(36.2 \%$ vs. $63.7 \%, \mathrm{p}=0.029)$ in a population of 570 patients [28]. Similarly, for Paciaroni et al., on a sample of 406, but without a significant difference [22]. Control of blood pressure reduces the risk of stroke by approximately $40 \%$ [42]. Hence, the need to implement a hypertension screening strategy to prevent its complications.

A history of diabetes (15.2\%), smoking (3.4\%) and alcohol $(27.8 \%)$ in our patients was lower than hypertension. However, Arnold et al. [28] reported a higher smoking prevalence $(38.7 \%)$ in patients with swallowing disorders, certainly related to lifestyle habits different from ours. Congolese people smoke less and consume alcohol more often [43, 44]. The prevalence of atherosclerotic lesions in diabetes and smoking people in Congo has already been reported [39]. It is mainly cardiovascular factors associated with the occurrence of stroke.

The history of stroke was found in $33.9 \%$ of our patients. This could explain a bilateral involvement of the corticobulbar bundle, so $72.9 \%$ of our patients had a bilateral pyramidal syndrome. The pseudobulbar syndrome has been frequently associated with swallowing disorders, preferentially cortical involvement $[27,28,33]$.

\subsection{Clinical Manifestations Associated with Swallowing Disorders}

Cough during feeding $(79.7 \%)$ and swallowing effort $(81.4 \%)$ were the main complaint of our patients as well as elsewhere [16, 40, 45]. Saliva accumulation in the mouth $(22 \%)$ and difficulty breathing during feeding $(5.1 \%)$ were less often reported. It was reported that salivary flow at rest would be significantly lower in stroke patients than in healthy subjects with $\mathrm{p}<0.01$ [46], so swallowing disorders could be justified by an accumulation of saliva due to a swallowing default.

Right hemiplegia was found in $18.6 \%$ of our patients, and left in $6.8 \%$. The pharyngeal and oesophageal muscles have asymmetric cortical distribution between the two hemispheres, suggesting the hypothesis of a "dominant" swallowing hemisphere more likely to cause swallowing disorders in stroke [8, 47-49].

In addition, left hemisphere involvement has been associated with a more extensive pre-pharyngeal phase of swallowing, and left hemisphere involvement with more severe pharyngeal dysfunctions, related to oral-facial apraxia $[10,50-52]$. However, the majority of our patients $(72.9 \%)$ had a bilateral pyramidal syndrome, easily explained by the pseudobulbar syndrome reported above.

The frequency of hemorrhagic stroke found in our study was $25.4 \%$ versus $74 \%$ for ischemic stroke. This predominant association between ischemic stroke and the onset of swallowing disorders was reported by several authors [13, 22, 32, 33].

\subsection{Evaluation of Local Feeding Protocol}

A nasogastric tube was placed in $92 \%$ of our patients. In five $(8.5 \%)$ patients, it was not, because swallowing disorders were judged to be minor. In developed countries, the use of the nasogastric tube is less frequent because other palliative measures are performed such as percutaneous endoscopic gastrostomy [28]. Moreover, nasogastric tube is kept less than three weeks because of its unpleasant nature, the risk of being torn off by the patient and thus of irritating esophagus or pharynx, and its impropriety during rehabilitation $[28,53]$. Percutaneous endoscopic gastrostomy, although possible in our context, is difficult to envisage because it causes to patients and family carers a sense of clinical severity, and also by the quality of care it requires and the cost relating to its implementation.

The time between diagnosis and nasogastric tube placement was $1.35 \pm 1$ [ 0 to 7 days]. This time is explained by the reluctance of the patient or his entourage on feeling of clinical worsening caused by nasogastric tube and also because the entourage were forced to obtain it outside the hospital because not available on site.

Our local feeding protocol was respected by only half of family carers. This for financial reasons and the presence of many family carers, interfering with the proper instruction of the health care team, too deficient in number. However, compliance or not with the local feeding protocol did not influence the occurrence of malnutrition or that of dehydration (respectively, $\mathrm{p}=0.58$ and 0.79 ).

\subsection{Patients Follow-up}

In our study, spontaneous recovery of swallowing disorders was observed in $3.39 \%$ of patients at one week, $12 \%$ at two weeks, $2.63 \%$ at one month and $11.42 \%$ at two months. No recovery was observed at three months. It should be noted that none of our patients received rehabilitation for their swallowing disorders. Typically, $50 \%$ of post-stroke swallowing disorders recover within two weeks, and $10 \%$ have sequelae beyond six months [28, 31, 32, 54]. Functional neuroimaging studies and neurophysiological studies using transcranial magnetic stimulation have shown that patients who have recovered their swallowing function will show an increase in activity in the perilesional cortical zones and in the unaffected hemisphere, at one and three months, suggesting reorganization of the unaffected motor cortex [5456]. At three months, none of our patients recovered their swallowing function. One study reported that, at three months, there was less regression of swallowing disorders, which would persist in $50.9 \%$ of patients discharged from hospital [28].

Complications were much more common during the first two weeks. At one week, the complications observed were mostly related to stroke. At two weeks, these were swallowing disorders-related complications due to delayed 
diagnosis and reluctance to place the nasogastric tube. Beyond, nursing staff and the family carers became more mobilized and adhered fully to the local feeding protocol. Home return home was made around the second month. Indeed, swallowing disorders were associated with a longer hospital stay [57].

The frequency of malnutrition in our study was $8.5 \%$ and of dehydration was $20.3 \%$. Swallowing disorders were reported to increase the risk of dehydration and/or malnutrition in stroke [15, 58-60]. Falseti et al. [13] reported a prevalence of $95.1 \%$ of patients with swallowing disorders who developed malnutrition with a significant difference compared to patients without swallowing disorders $(p=0.023)$. Neurological swallowing disorders would increase the risk of malnutrition by 2.43 [61].

We recorded $54.2 \%$ of deaths. Arnold et al. reported a death rate of $13.6 \%$ [24]. Neurological swallowing disorders were correlated with a high risk of mortality $[57,62]$. In our study, one-month mortality was $5.26 \%$ and three-year mortality was $13.6 \%$. Huang et al. followed patients with swallowing disorders over one year and reported a death rate of $45 \%$ [33].

In Congo, management of swallowing disorders remains a serious public health problem. The lack of adequate care, including palliative methods other than the nasogastric tube, as well as the delayed diagnosis and placement of the nasogastric tube, would justify the importance of death [32, 54]. Percutaneous endoscopic gastrostomy is more effective than the nasogastric tube in improving the nutritional status of patients with post-stroke swallowing disorders, which instead deteriorating nutritional status [63].

In our study, $34.4 \%$ of patients died at home for an unknown cause. As explained above, the relative frequency of home-based deaths could be explained by home-based hospitalization that is not framed in our context and the exhaustion of family carers during follow-up, including the non-compliance with the local feeding protocol. Deaths recorded during hospitalization were due to $15.62 \%$ bronchopneumopathy and $12.50 \%$ false roads. Pulmonary complications are relatively common in patients with swallowing disorders $[28,64]$.

\section{Conclusion}

Swallowing disorders are a frequent and serious complication of stroke. Early detection and appropriate care should be systematic and included in service protocols. The DePippo test is useful for its detection. The mean delay of diagnosis is around 6 days, that explain some complications observed in relation with swallowing disorders such as dehydration, malnutrition and, bronchopneumopathy which is one of lead cause of death after stroke in Africa. Improving earlier diagnosis and management of swallowing disorders would rightly avoid its fatal outcome.

\section{Acknowledgements}

The authors thank the patients or their parents who participated in this study.

\section{Conflicts of Interest}

The authors have no financial relationship relevant to this article. They have no conflicts of interest.

\section{References}

[1] Bourazza A, Kerouache A, Reda R, Mounach J, Mosseddaq R. [Meningovascular syphilis: Study of five cases]. Rev. Neurol. (Paris) 2008;164(4):369-73.

[2] Adeloye D. An estimate of the incidence and prevalence of stroke in Africa: a systematic review and meta-analysis. PLoS One2014;9(6):e100724. doi: 10.1371/journal.pone.0100724.

[3] Adoukonou TA, Vallat JM, Joubert J, Macian F, Kabore R, Magy L, et al. [Management of stroke in sub-Saharan Africa: Current issues]. Rev. Neurol. (Paris) 2010;166(11):882-93.

[4] Gombet T, Ossou-Nguiet PM, Ampion MO, Ellenga-Mbolla BF, Bandzouzi-Ndamba B, Matali E, et al. Facteurs de mortalité des accidents vasculaires cérébraux au CHU de Brazzaville. Rev. Neurol. (Paris) 2012;168:A87-A8.

[5] Mann G, Hankey GJ. Initial clinical and demographic predictors of swallowing impairment following acute stroke. Dysphagia. 2001;16(3):208-15.

[6] Martino R, Foley N, Bhogal S, Diamant N, Speechley M, Teasell R. Dysphagia after stroke: incidence, diagnosis, and pulmonary complications. Stroke 2005;36(12):2756-63.

[7] Lakshminarayan K, Tsai AW, Tong X, Vazquez G, Peacock $\mathrm{JM}$, George MG, et al. Utility of dysphagia screening results in predicting poststroke pneumonia. Stroke. 2010;41(12):2849-54.

[8] Singh S, Hamdy S. Dysphagia in stroke patients. Postgrad. Med. J. 2006;82(968):383-91.

[9] Walter U, Knoblich R, Steinhagen V, Donat M, Benecke R, Kloth A. Predictors of pneumonia in acute stroke patients admitted to a neurological intensive care unit. J. Neurol. 2007;254(10):1323.

[10] Steinhagen V, Grossmann A, Benecke R, Walter U. Swallowing disturbance pattern relates to brain lesion location in acute stroke patients. Stroke. 2009;40(5):1903-6.

[11] Ossou-Nguiet P, Ellenga-Mbolla B, Odzebe A, Otiobanda G, Gankama T, Obondzo-Aloba K, et al. Impact of urinary tract and pulmonary infection on mortality after intracerebral hemorrhage in Brazzaville. World J. Neurosci. 2013;3(04):246.

[12] Hilker R, Poetter C, Findeisen N, Sobesky J, Jacobs A, Neveling $\mathrm{M}$, et al. Nosocomial pneumonia after acute stroke: implications for neurological intensive care Medicine. Stroke 2003;34(4):975-81.

[13] Falsetti P, Acciai C, Palilla R, Bosi M, Carpinteri F, Zingarelli $\mathrm{A}$, et al. Oropharyngeal dysphagia after stroke: incidence, diagnosis, and clinical predictors in patients admitted to a neurorehabilitation unit. J. Stroke Cerebrovasc. Dis. 2009;18(5):329-35. 
[14] Runions S, Rodrigue N, White C. Practice on an acute stroke unit after implementation of a decision-making algorithm for dietary management of dysphagia. J. Neurosci. Nurs. 2004;36(4):200.

[15] Capet C, Delaunay O, Idrissi F, Landrin I, Kadri N. [Swallowing disorders in the elderly: recognizing risk factors for early management]. NPG Neurologie - PsychiatrieGériatrie2007;7(40):15-23.

[16] Carnaby-Mann G, Lenius K, Crary M. Update on assessment and management of dysphagia post stroke. Northeast Florida Med. 2007;58(2):31-4.

[17] Badve MS, Zhou Z, Anderson CS, Hackett ML. Effectiveness and safety of antibiotics for preventing pneumonia and improving outcome after acute stroke: systematic review and meta-analysis. J. Stroke Cerebrovasc. Dis. 2018 Aug6. pii:SS1052-3057(18)30368-9.

[18] Morrell K, Hyers M, Stuchiner T, Lucas L, Schwartz K, Mako $\mathrm{J}$, et al. Telehealth stroke dysphagia evaluation is safe and effective. Cerebrovasc. Dis. 2017;44(3-4):225-31.

[19] Nordio S, Innocenti T, Agostini M, Meneghello F, Battel I. The efficacy of telerehabilitation in dysphagic patients: a systematic review. ActaOtorhinolaryngol. Ita. 2018;38(2):7985 .

[20] Huang YC, Hsu TW, Leong CP, Hsieh HC, Lin WC. Clinical effects and differences in neural function connectivity revealed by MRI in subacute hemispheric and brainstem infarction patients with dysphagia after swallowing therapy. Front. Neurosci. 2018;12:488.

[21] DePippo KL, Holas MA, Reding MJ. Validation of the 3-oz water swallow test for aspiration following stroke. Arch. Neurol. 1992;49(12):1259-61.

[22] Paciaroni M, Mazzotta G, Corea F, Caso V, Venti M, Milia P, et al. Dysphagia following Stroke. Eur. Neurol. 2004;51(3):162-7.

[23] Clark WM, Wissman S, Albers GW, Jhamandas JH, Madden KP, Hamilton S. Recombinant tissue-type plasminogen activator (Alteplase) for ischemic stroke 3 to 5 hours after symptom onset. The ATLANTIS Study: a randomized controlled trial. Alteplase Thrombolysis for Acute Noninterventional Therapy in Ischemic Stroke. Jama. 1999;282(21):2019-26.

[24] Audebert HJ, Rott MM, Eck T, Haberl RL. Systemic inflammatory response depends on initial stroke severity but is attenuated by successful thrombolysis. Stroke. 2004;35(9):2128-33.

[25] BaroniAF, Fàbio SR, Dantas RO. Risk factors for swallowing dysfunction in stroke patients. Arg. Gastroenterol. 2012;49(2):118-24.

[26] Mackay LE, Morgan AS, Bernstein BA. Swallowing disorders in severe brain injury: Risk factors affecting return to oral intake. Arch. Phys. Med. Rehabil. 1999;80(4):365-71.

[27] Langdon PC, Lee AH, Binns CW. Dysphagia in acute ischaemic stroke: severity, recovery and relationship to stroke subtype. J. Clin. Neurosci. 2007;14(7):630-4.

[28] Arnold M, Liesirova K, Broeg-Morvay A, Meisterernst J, Schlager M, Mono M-L, et al. Dysphagia in acute stroke: incidence, burden and impact on clinical outcome. PloS one.

\section{6;11(2):e0148424.}

[29] Crary MA, Carnaby GD, Sia I, Khanna A, Waters MF. Spontaneous Swallowing Frequency Has Potential to Identify Dysphagia in Acute Stroke. Stroke. 2013;44(12):3452-7.

[30] SørensenRT, Rasmussen RS, Overgaard K, Lerche A, Johansen AM, Lindhardt T. Dysphagia screening and intensified oral hygiene reduce pneumonia after stroke. J. Neurosci. Nurs. 2013;45(3):139-46.

[31] Kidd D, Lawson J, Nesbitt R, MacMahon J. The natural history and clinical consequences of aspiration in acute stroke. QJM 1995;88(6):409-13.

[32] Ickenstein GW, Hohlig C, Prosiegel M, Koch H, Dziewas R, Bodechtel U, et al. Prediction of outcome in neurogenic oropharyngeal dysphagia within 72 hours of acute stroke. J. Stroke Cerebrovasc. Dis. 2012;21(7):569-76.

[33] de AlencarNunes MC, Jurkiewicz AL, Santos RS, Furkim AM, Massi G, Pinto GSA, et al. Correlation between brain injury and dysphagia in adult patients with stroke. Int. Arch. Otorhinolaryngol. 2012;16(03):313-21.

[34] Al-Khaled M, Matthis C, Binder A, Mudter J, Schattschneider J, Pulkowski U, et al. Dysphagia in patients with acute stroke: early dysphagia screening may reduce stroke-related pneumonia and improve stroke outcome. Cerebrovasc. Dis. 2016;42(1-2):81-9.

[35] Desport J-C, Fayemendy P, Jésus P, Salle J-Y. [Management of swallowing disorders]. Nutr. Clin. Metab. 2014;28(3):2214.

[36] Pouderoux P. Troubles de la déglutition: étiologies et prise en charge. Hépato-Gastro \& Oncologie Digestive. 1999;6(4):24757.

[37] Huang K-L, Liu T-Y, Huang Y-C, Leong C-P, Lin W-C, Pong Y-P. Functional Outcome in Acute Stroke Patients with Oropharyngeal Dysphagia after Swallowing Therapy. J. Stroke Cerebrovasc. Dis. 2014;23(10):2547-53.

[38] Ossou-Nguiet PM, Gombet TR, OssilAmpion M, Otiobanda GF, Obondzo-Aloba K, Bandzouzi-Ndamba B. [Gender and stroke in Brazzaville]. Rev. Epidémiol. Sante2014;62(1):7882 .

[39] Ikama MS, Nsitou BM, Makani J, Nkalla-Lambi M, PassiLouamba C. [Hypertension and rate control in Brazzaville (Congo): Place of ambulatory blood pressure monitoring (ABPM)]. Annal. Cardiol. Angéiol. 2015;64(2):76-80.

[40] Medeiros GCd, Sassi FC, Mangilli LD, Zilberstein B, Andrade CRFd. Clinical dysphagia risk predictors after prolonged orotracheal intubation. Clinics. 2014;69:8-14.

[41] Gombet TR, Ossou-Nguiet PM, Gankama TN, EllengaMbolla BF, Otiobanda GF, Obondzo-Aloba K, et al. Hypertension and intracerebral hemorrhage in Brazzaville. World J. Cardiovasc. Dis. 2013;3(09):523.

[42] Lemogoum D, Degaute J-P, Bovet P. Stroke Prevention, Treatment, and Rehabilitation in Sub-Saharan Africa. Am. J. Prev. Med. 2005;29(5):95-101.

[43] Gassaye D, Bossali F, Atipo-Ibara B, Ahoui CR, Mouele MD, Ibara JR. Prévalence de la consommation d'alcool dans la ville de Brazzaville en 2014. Journal Africain d'HépatoGastroentérologie 2015;9(4):160-2. 
[44] Kimbally K, G, Voumbo Y, Gombet T, Ikama M, S , Bolanda $\mathrm{J}$, Gokaba C, et al. Etude de la prévalence de la consommation de l'alcool et du tabac à Brazzaville. Cardiologie Tropicale $2008 ; 129(33)$.

[45] Salle J-Y, Lissandre J-P, Morizio A, Bouthier-Quintard F, Desport J-C. Dépistage et prise en charge des troubles de la déglutition chez les personnes âgées. In : Traité de nutrition de la personne âgée. Springer (Paris) 2009. p. 221-7.

[46] Kim IS, Han TR. Influence of Mastication and Salivation on Swallowing in Stroke Patients. Arch. Phys. Med. Rehabil. 2005;86(10):1986-90.

[47] Saito T, Hayashi K, Nakazawa H, Ota T. Clinical characteristics and lesions responsible for swallowing hesitation after acute cerebral infarction. Dysphagia 2016;31(4):567-73.

[48] Hamdy S, Aziz Q, Rothwell JC, Crone R, Hughes D, Tallis $\mathrm{RC}$, et al. Explaining oropharyngeal dysphagia after unilateral hemispheric stroke. Lancet (London) 1997;350(9079):686-92.

[49] Teismann IK, Dziewas R, Steinstraeter O, Pantev C. Timedependent hemispheric shift of the cortical control of volitional swallowing. Hum. Brain Mapp. 2009;30(1):92-100.

[50] Moon HI, Yoon SY, Jeong YJ, Cho TH. Lesions responsible for delayed oral transit time in post-stroke dysphagia. Dysphagia 2018;33(3):321-8.

[51] Daniels SK, Brailey K, Foundas AL. Lingual discoordination and dysphagia following acute stroke: analyses of lesion localization. Dysphagia. 1999;14(2):85-92.

[52] Kwon M, Lee JH, Kim JS. Dysphagia in unilateral medullary infarction: lateral vs medial lesions. Neurology 2005;65(5):714-8.

[53] Flamand-Roze C, Roze E, Denier C. [Language and swallowing disorders in acute stroke patients: Tools and early management]. Rev. Neurol. (Paris) 2012;168(5):415-24.

[54] Barritt AW, Smithard DG. Role of cerebral cortex plasticity in the recovery of swallowing function following dysphagic stroke. Dysphagia 2009;24(1):83-90.
[55] Hamdy S, Aziz Q, Rothwell JC, Power M, Singh KD, Nicholson DA, et al. Recovery of swallowing after dysphagic stroke relates to functional reorganization in the intact motor cortex. Gastroenterology. 1998;115(5):1104-12.

[56] Teismann IK, Suntrup S, Warnecke T, Steinstrater O, Fischer $\mathrm{M}$, Floel A, et al. Cortical swallowing processing in early subacute stroke. BMC Neurology 2011;11:34.

[57] Smithard DG, O'Neill PA, Parks C, Morris J. Complications and outcome after acute stroke. Does dysphagia matter? Stroke 1996;27(7):1200-4.

[58] González-Fernández M, Ottenstein L, Atanelov L, Christian AB. Dysphagia after stroke: an overview. Curr. Phys. Med. Rehabil. Rep. 201;1(3):187-96.

[59] Le Fort M, Hamon P, Mathé J-F, Perrouin-Verbe B. Typologie analytique et fonctionnelle de troubles de la déglutition selon l'étiologie neurologique ou neuromusculaire de 153 patients adressés à la consultation spécialisée du CHU de Nantes. Ann. Phys. Rehabil. Med. 2011;54:e31.

[60] Altman KW, Yu GP, Schaefer SD. Consequence of dysphagia in the hospitalized patient: impact on prognosis and hospital resources. Arch. Otolaryngol. Head Neck Surg. 2010;136(8):784-9.

[61] Foley NC, Martin RE, Salter KL, Teasell RW. A review of the relationship between dysphagia and malnutrition following stroke. J Rehabil Med. 2009;41(9):707-13.

[62] Collaboration FT. Poor nutritional status on admission predicts poor outcomes after stroke: observational data from the FOOD trial. Stroke. 2003;34(6):1450-6.

[63] Hamidon BB, Abdullah SA, Zawawi MF, Sukumar N, Aminuddin A, Raymond AA. A prospective comparison of percutaneous endoscopic gastrostomy and nasogastric tube feeding in patients with acute dysphagic stroke. Med. J. Malaysia 2006;61(1):59-66.

[64] Lim K-B, Lee H-J, Lim S-S, Choi Y-I. Neuromuscular electrical and thermal-tactile stimulation for dysphagia caused by stroke: A randomized controlled trial. J. Rehabil. Med. 2009;41(3):174-8. 